\title{
Slotted Aloha with Priorities and Random Power
}

\author{
E. Altman ${ }^{1, \star}$, D. Barman ${ }^{2, \star \star}$ A. Benslimane, and R. El Azouzi ${ }^{3}$ \\ 1 INRIA, BP93, 06902 Sophia-Antipolis, France \\ eitan.altman@sophia.inria.fr \\ 2111 Cummington Street, Dept. of Computer Science, \\ Boston University, Boston, MA 02215, USA \\ dhiman@cs.bu.edu \\ 3 LIA/CERI, Université d'Avignon, Agroparc, BP 1228, 84911, Avignon, France \\ \{benslimane, rachid.elazouzi\}@univ-avignon.fr
}

\begin{abstract}
We study distributed choice of retransmission probabilities in slotted Aloha under power differentiation. We consider random transmission powers and further study the role of priorities (through power control) given either to new arriving packets or to backlogged ones. We study both the cooperative team problem in which a common objective is jointly optimized as well as the noncooperative game problem. We show that the new proposed schemes not only improve the average performances considerably but are also able in some cases to eliminate the bi-stable nature of the slotted Aloha.
\end{abstract}

\section{Introduction and Problem Formulation}

Aloha [1] and slotted Aloha [1] have long been used as random distributed medium access protocols for radio channels. They are used in satellite networks and cellular telephone networks for the sporadic transfer of data packets. In these protocols, packets are transmitted sporadically by various users. If packets are sent simultaneously by more than one user then they collide. After a packet is transmitted, the transmitter receives the information on whether there has been a collision (and retransmission is needed) or whether it has been well received. All colliding packets are assumed to be corrupted which get backlogged and are retransmitted after some random time. We focus on the slotted Aloha [5] in which time is divided into units. At each time unit a packet may be transmitted, and at the end of the time interval, the sources get the feedback on whether there was zero, one or more transmissions (collision) during the time slot. A packet that arrives at a source is immediately transmitted. We introduce three new schemes in which multiple power levels are used for transmission. When several packets are sent simultaneously, one of them can often be successfully

\footnotetext{
* Partially supported by the Euro-NGI network of excellence.

${ }^{\star \star}$ Partially supported by INRIA through the ARC PRIXNET.
} 
received due to the power capture effect. We assume that the packet with the largest received power captures the channel [7, 10, 12, 13]; if two or more packets are transmitted simultaneously with the same power, we assume that neither one can be captured. In addition to the power diversity, already proposed in [7,10,12,13, we consider differentiation between new packets and backlogged packets allowing prioritization of one or the other in terms of transmitted power. We study and compare (1) a scheme with power diversity and without prioritization in transmission or retransmission; (2) a scheme in which a new packet is transmitted with the lowest power, and backlogged packets are transmitted at a random power selected among $N$ larger distinct levels; (3) a scheme in which a new packet is transmitted with the highest power, and backlogged packets are transmitted at a random power selected among $N$ lower distinct levels; and (4) standard slotted Aloha.

We first consider the optimal selection of transmission probabilities for the various schemes so as to maximize the throughput or minimize the expected delay. We discover that in heavy load, the optimality is obtained at the expense of huge expected delay of backlogged packets (EDBP). We therefore consider also the alternative objective of minimizing the EDBP. We also solve the multicriteria problem. We show that the new schemes not only improve the average performances considerably but also improve the stability performance.

In addition to the global optimization, we study the game problem in which each mobile chooses its transmission probability selfishly so as to optimize its individual objective. We show that the power diversity and the prioritization profit to mobiles also in this competitive scenario.

Various game formulations of slotted aloha with a single power have been derived and studied in $3,4,6,8,9$, for the non-cooperative choice of transmission probabilities. Several papers study slotted aloha with power diversities but without differentiating between transmitted and backlogged packets, and without the game formulation [7, 10, 12, 13. We consider a central receiver and $m$ sources without buffer. We assume a perfect capture model where a successful capture of a packet at the receiver occurs when the power level (among $N$ different levels) selected for this packet is greater than those of all other packets transmitted in the same slot.

We use a Markovian model extending [5-Sec. 4.2.2]. Packet arrivals to sources, independently of each other, follow Bernoulli process with parameter $q_{a}$. As long as there is a packet at a source (i.e. it has not been successfully transmitted) new packets to that source are blocked and lost 1 A backlogged packet at source $i$ is retransmitted with probability $q_{r}^{i}$ that does not change with time. Since sources are symmetric, we shall further restrict to finding a symmetric optimal solution (i.e., $q_{r}^{i}=q, \forall i$ ).

We shall use as the state of the system the number of backlogged nodes (or equivalently, of backlogged packets) at the beginning of a slot, and denote it

$\overline{1}$ This assumption is equivalent to saying that a source does not generate new packets as long as a previous packet is not successfully transmitted. 
frequently with $n$. For any choice of $q_{r}^{i} \in(0,1]$, the state process is a Markov chain that contains a single ergodic chain. Let $\mathbf{q}_{r}$ be the vector of retransmission probabilities for all users (whose $j$ th entry is $q_{r}^{j}$ ). Let $\pi\left(\mathbf{q}_{r}\right)$ be the corresponding vector of steady state probabilities where its $n$th entry, $\pi_{n}\left(\mathbf{q}_{r}\right)$, denotes the probability of $n$ backlogged nodes. When all entries of $\mathbf{q}_{r}$ are the same, say $q$, we shall write $\pi(q)$ instead of $\pi\left(\mathbf{q}_{r}\right)$.

Assume that there are $n$ backlogged packets, and all use the same value $q_{r}$ as retransmission probability. Let $Q_{r}(i, n)$ be the probability that $i$ out of the $n$ backlogged packets retransmit at the slot. Let $Q_{r}(1,0)=0$. Then $Q_{r}(i, n)=$ $\left(\begin{array}{c}n \\ i\end{array}\right)\left(1-q_{r}\right)^{n-i} q_{r}^{i}$. Let $Q_{a}(i, n)$ be the probability that $i$ unbacklogged nodes transmit packets in a given slot (i.e. that $i$ arrivals occurred at nodes without backlogged packets). Let $Q_{a}(1, m)=0$. Then $Q_{a}(i, n)=\left(\begin{array}{c}m-n \\ i\end{array}\right)\left(1-q_{a}\right)^{m-n-i} q_{a}^{i}$.

\section{Team Problem}

In this section we propose and analyze three different schemes. We observe that standard slotted Aloha is a special case of these proposed schemes.

Scheme 1: Random power levels without priority: A mobile transmits a packet (new or backlogged) using one of $N$ distinct available power levels uniformly chosen and that does not depend on the type of packet. In case all nodes use the same value $q$ and $q_{r}$, the transition probabilities of the Markov chain is given in $[2]$.

Scheme 2: Retransmission with more power: A backlogged packet retransmits with a power from $N$ different distinct levels. A new arrival packet uses a lower power than any one these $N$ levels. The random power levels are chosen uniformly. Successful capture occurs if one of the backlogged packet transmits with a power level larger than that chosen by all others transmitters or if a single new arrival occurs and there is no retransmission attempt of any backlogged packet. The transition matrix is given in [2].

Scheme 3 : Retransmission with less power: A new transmitted packet has the highest power. Backlogged packets attempt retransmissions with a random power choice among $N$ distinct lower power levels. The random power levels are chosen uniformly. The transition matrix is given in [2].

Performance Metrics. Denote by $\pi_{n}(q)$ the equilibrium probability that the network is in state $n$ and $P(q)$ the transition matrix of a scheme. Then we have the equilibrium state equations:

$$
\left\{\begin{array}{c}
\pi(q)=\pi(q) P(q), \\
\pi_{n}(q) \geq 0, n=0, \ldots, m \\
\sum_{n=0}^{m} \pi_{n}(q)=1 .
\end{array}\right.
$$

The average number of backlogged packets is

$$
S(q)=\sum_{n=0}^{m} \pi_{n}(q) n .
$$


The system throughput (i.e. the sample time average of the number of packets that are successfully transmitted) is given almost surely by the constant,

$$
\operatorname{thp}(q)= \begin{cases}\sum_{n=1}^{m} \pi_{n}(q)\left[Q_{a}(0, n) \sum_{j=1}^{n} Q_{r}(j, n) A_{j}+Q_{a}(1, n) \sum_{j=0}^{n} Q_{r}(j, n) A_{j+1}+\right. & \\ \left.\sum_{i=2}^{m-n} Q_{a}(i, n) \sum_{j=0}^{n} Q_{r}(j, n) A_{i+j}\right]+\pi_{0}(q) Q_{a}(1,0) & \text { scheme1 } \\ \sum_{n=1}^{m} \pi_{n}(q)\left[Q_{a}(1, n) Q_{r}(0, n)+Q_{r}(1, n)+\right. & \\ \left.\sum_{j=2}^{n} Q_{r}(j, n) A_{j}\right]+\pi_{0}(q) Q_{a}(1,0) & \text { scheme2 } \\ \sum_{n=0}^{m} \pi_{n}(q)\left[Q_{a}(1, n)+Q_{r}(0, n)\left\{Q_{r}(1, n)+\sum_{j=2}^{n} Q_{r}(j, n) A_{j}\right\}\right] & \text { scheme3 }\end{cases}
$$

where $A_{k}$ is the probability of successful transmission among $k \geq 2$ packets and is given by $A_{k}=k \sum_{l=0}^{N-1} X_{N-l}\left(1-\sum_{i=N-l}^{N} X_{i}\right)^{k-1}, A_{0}=0, A_{1}=1$ and $X_{i}$ is the probability that a packet (new arrival or backlogged) will choose power level $i$ for retransmission. The throughput satisfies

$$
\operatorname{thp}(q)=q_{a} \sum_{n=0}^{m} \pi_{n}(q)(m-n)=q_{a}(m-S(q)) .
$$

Eq.(3) equals to the expected number of arrivals per slot (which actually enter the system), and to the expected number of departures per slot. The expected delay of transmitted packets $\mathrm{D}$, is defined as the average time that a packet takes from its source to the receiver. Applying Little's result, this is given by

$$
D(q)=1+\frac{S(q)}{\operatorname{thp}(q)}=1+\frac{S(q)}{q_{a}(m-S(q))}
$$

The first term accounts for the first transmission from the source. Combining the last equality in (3) with (4) it follows that maximizing the global throughput is equivalent to minimizing the average delay of transmitted packets. We shall therefore restrict in our numerical investigation to maximization the throughput. However, we shall consider the delay of backlogged packets (EDBP) as yet another objective to minimize.

Performance Measures for Backlogged Packets. The throughput of the backlogged packets for each scheme is given by, $\operatorname{th} p^{c}=\operatorname{th} p(q)-\Delta$ where $\Delta$ is given by:

$$
\Delta= \begin{cases}\sum_{n=0}^{m} \sum_{i=1}^{m-n} \sum_{j=0}^{n}\left\{\frac{i}{i+j} Q_{a}(i, n) Q_{r}(j, n) A_{i+j}\right\} \pi_{n}(q) & \text { scheme } 1 \\ \sum_{n=0}^{m} Q_{a}(1, n) Q_{r}(0, n) \pi_{n}(q), & \text { scheme 2 } \\ \sum_{n=0}^{m} Q_{a}(1, n) \pi_{n}(q) & \text { scheme 3 }\end{cases}
$$


The $\operatorname{EDBP} D^{c}$ is the average time, in slots, that a backlogged packet takes to go from the source to receiver. Applying Little's Theorem, the expected delay of packets that arrive and become backlogged is given by:

$$
D^{c}(q)=1+S(q) / \operatorname{thp}^{c}(q)
$$

The team problem is therefore given as the solution of

$$
\max _{q} \text { objective }(q) \text { s.t. }\left\{\begin{array}{c}
\pi(q)=\pi(q) P(q), \\
\pi_{n}(q) \geq 0, n=0, \ldots, m \\
\sum_{n=0}^{m} \pi_{n}(q)=1 .
\end{array}\right.
$$

The solution can be obtained by computing recursively the steady state probabilities, as in Problem 4.1 in [5], and thus obtain an explicit expression for thp $(q)$ as a function of $q$.

Stability. Slotted aloha is known to have a bi-stable behavior, and we shall check whether this is also the case in our new schemes. We try to find the drift, $D_{n}$ in state $n$ which is the difference between the expected number of new arrivals and new successful departures over a slot time, starting in state $n$ [5].

$$
D_{n}=(m-n) q_{a}-P_{\text {succ }}
$$

where the probability of a successful transmission, $P_{\text {succ }}$ for each scheme is given in [2]. For standard slotted aloha it has been observed (see [5]) that there are three equilibria, where an equilibrium is defined as a state $n$ in which the arrival rate $(m-n) q_{a}$ equals the departure rate $P_{\text {succ }}$. Moreover, among those three, the two extreme ones (the one corresponding to the smallest and to the largest state) are stable $2 \mathrm{~A}$ bi-stable situation is undesirable since it could mean that the system spends long time in each of the stable equilibria including in the one with large $n$ (low throughput and large delays).

\section{Game Problem}

This formulation is of interest in decentralized scenarios where mobiles may not be controlled by a centralized entity. The equilibrium concept then replaces the optimality concept from the team problem. It possesses a robustness property: at equilibrium, no mobile has incentive to deviate.

For a given policy vector $\mathbf{q}_{\mathbf{r}}$ of retransmission probabilities for all users (whose $j$ th entry is $\left.q_{r}^{j}\right)$, define $\left(\left[\mathbf{q}_{\mathbf{r}}\right]^{-i}, q_{r}^{i}\right)$ to be a retransmission policy where user $j$ retransmits at a slot with probability $q_{r}^{j}$ for all $j \neq i$ and where user $i$ retransmits with probability $q_{r}^{i}$ and $\left[\mathbf{q}_{\mathbf{r}}\right]^{-i}$ represents the policy vector without user $i$. Each user $i$ seeks to maximize his own objective $_{i}(\mathbf{q})$. We then seek for a symmetric

$\overline{{ }^{2} \text { Recall that }}$ an equilibrium is stable if the drift corresponding to a small deviation (increasing or decreasing $n$ ) from the equilibrium is in the direction opposite to the deviation. 
equilibrium policy $\mathbf{q}_{\mathbf{r}}^{*}=\left(q_{r}, q_{r}, . ., q_{r}\right)$ such that for any $i$ and any retransmission probability $q_{r}^{i}$,

$$
\text { objective }_{i}\left(\mathbf{q}_{\mathbf{r}}^{*}\right) \geq \text { objective }_{i}\left(\left[\mathbf{q}_{\mathbf{r}}^{*}\right]^{-i}, q_{r}^{i}\right)
$$

where the objective function is the throughput or minus the expected delay. Since we restrict to symmetric $\mathbf{q}_{\mathbf{r}}^{*}$, we shall also identify it with the actual transmission probability (which is the same for all users). Next we show how to obtain an equilibrium. We note that due to symmetry, to see whether $\mathbf{q}_{\mathbf{r}}^{*}$ is an equilibrium it suffices to check (7) for a single player. We shall thus assume that there are $m+1$ users all together, and that the first $m$ users retransmit with a given probability $\mathbf{q}_{\mathbf{r}}{ }^{-(m+1)}=\left(q^{o}, . ., q^{o}\right)$ and user $m+1$ retransmits with probability $q_{r}^{(m+1)}$. Define the set

$$
\mathcal{Q}^{m+1}\left(\mathbf{q}_{\mathbf{r}}\right)=\arg \max _{q_{r}^{(m+1)} \in[\epsilon, 1]}\left(\text { objective }_{m+1}\left(\left[\mathbf{q}_{\mathbf{r}}\right]^{-(m+1)}, q_{r}^{(m+1)}\right)\right)
$$

where $\mathbf{q}_{\mathbf{r}}$ denotes the policy where all users retransmit with probability $q_{r}^{o}$, and where the maximization is taken with respect to $q_{r}^{(m+1)}$. Then $q_{r}^{*}$ is a symmetric equilibrium if $q_{r}^{*} \in \mathcal{Q}_{r}^{m+1}\left(q_{r}^{*}\right)$. To compute the performance measures of interest objective $e_{m+1}\left(\left[\mathbf{q}_{\mathbf{r}}\right]^{-i}, q_{r}^{i}\right)$, we introduce again a Markov chain with a two dimensional state. The first component corresponds to the number of backlogged packets among users $1, \ldots, m$, and the second is the number of backlogged packets (either 1 or 0 ) of user $m+1$. The various schemes considered are the same as in the team problem.

Performance Metrics. In the game problem, the average number of backlogged packets of source $m+1$ is given by:

$$
S_{m+1}\left(\left[\mathbf{q}_{\mathbf{r}}\right]^{-(m+1)}, q_{r}^{m+1}\right)=\sum_{n=0}^{m} \pi_{n, 1}\left(\left[\mathbf{q}_{\mathbf{r}}\right]^{-(m+1)}, q_{r}^{(m+1)}\right)
$$

and the average throughput of user $m+1$ is given by

$$
t h p_{m+1}\left(\left[\mathbf{q}_{\mathbf{r}}\right]^{-(m+1)}, q_{r}^{(m+1)}\right)=q_{a} \sum_{n=0}^{m} \pi_{n, 0}\left(\left[\mathbf{q}_{\mathbf{r}}\right]^{-(m+1)}, q_{r}^{(m+1)}\right)
$$

Hence the expected delay of transmitted packets of user $m+1$ is given by

$$
\left.D_{m+1}\left(\mathbf{q}_{\mathbf{r}}\right]^{-(m+1)}, q_{r}^{m+1}\right)=1+\frac{S_{m+1}\left(\left[\mathbf{q}_{\mathbf{r}}\right]^{-(m+1)} q_{r}^{m+1}\right)}{t h p_{m+1}\left(\left[\mathbf{q}_{\mathbf{r}}\right]^{-(m+1)}, q_{r}^{m+1}\right)}
$$

Let us denote by $t h p_{m+1}^{c}$ the throughput of backlogged packets (i.e. of the packets that arrive and become backlogged) at source $m+1$ :

$$
t h p_{m+1}^{c}\left(\mathbf{q}_{m+1}\right)=\sum_{n=0}^{m} \sum_{n^{\prime}=0}^{m} P_{(n, 0),\left(n^{\prime}, 1\right)}\left(\mathbf{q}_{m+1}\right) \pi_{n, 0}\left(\mathbf{q}_{m+1}\right)
$$

Thus, the expected delay of backlogged packets at source $m+1$, is given by

$$
D_{m+1}^{c}\left(\mathbf{q}_{m+1}\right)=1+S_{m+1}\left(\mathbf{q}_{m+1}\right) / t h p_{m+1}^{c}\left(\mathbf{q}_{m+1}\right)
$$




\section{Numerical Investigation}

In Figure 1 (a) and (b), we plot the throughput and EDBP for all schemes under the objective of maximizing the global throughput for $m=20$ and $N=$ 5. A general observation is that all new schemes outperform standard Aloha (Figure 1 (a)). The throughput of all the new schemes are comparable under all values of load. The result of maximizing throughput on the EDBP is plotted in Figure 1(b). All the schemes outperform standard Aloha but perform very bad at heavy load. We observe in Fig [1(b) that under low and moderate load, scheme 2 performs the best in minimizing EDBP as scheme 2 prioritizes the backlogged packets. Scheme 1 performs better than scheme 3 by prioritizing a fraction of backlogged packets while scheme 3 gives no priority to backlogged packets. The optimal retransmission probability in scheme 2 is higher than in scheme 1 and standard aloha which explains the best performance scheme 2 in terms of EDBP ([3]) and higher EDBP in scheme 1 and standard aloha.

Inspite of higher optimal retransmission probability in scheme 3 (compared to scheme 1 and standard aloha), scheme 3 experiences higher EDBP compared to scheme 2. In scheme 3, the new transmitted packets have the highest power and higher retransmission probability doesn't affect the successful transmission of new packets implying insensitivity of system's throughput to retransmission probabilities and resulting in dramatic increases in the EDBP and the best performances in the throughput and expected delay of transmitted packets, EDTP. Morever, in heavy traffic or when the number of mobiles increases, the throughput in scheme 3 is exactly the arrival probability: as the system is more congested, and the new arrivals have more priority, the steady probability is then given by $\pi_{m-1} \approx 1$. Hence, only the new packet arrived at free mobile, can be successfully transmitted.

Minimizing EDBP. On maximizing the global throughput we observed a huge EDBP under all schemes in heavy load which may be detrimental to many appli-

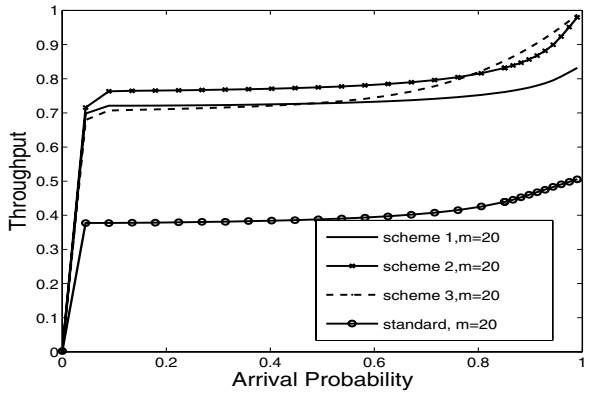

(a)

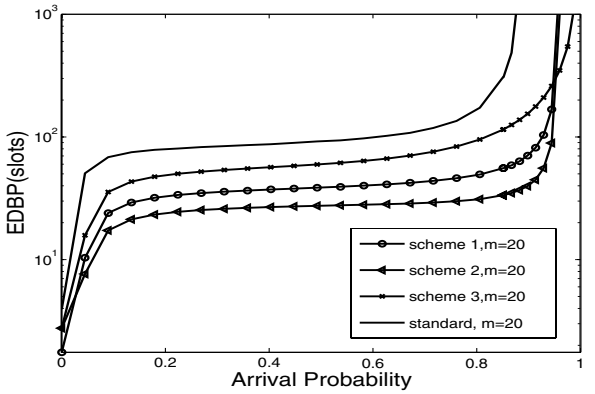

(b)

Fig. 1. (a) and (b) show the throughput and EDBP. The objective under all the schemes is to maximize the throughput. The number of mobiles is 20 and the number of levels is 5 
cation. We thus investigate directly the problem of minimizing EDBP and its impact on the throughput performance. We find in particular that throughput performance in the new schemes improves considerably with respect to standard aloha even when standard aloha uses the previous objective of maximizing throughput.

In Figure 2] we plot the performance of the various schemes for $m=20$. Part (a) considers the impact on the throughput while minimizing the EDBP. Furthermore, all three schemes outperform standard aloha even when the latter uses throughput maximization as optimization objective. We observe that the performance of scheme 3 , in contrast to other schemes, is insensitive to the choice of the objective optimization (throughput or EDBP), which explains the high throughput and dramatic increases in the EDBP ( see previous paragraph). Thus scheme 3 is very inefficient for many applications using the real time connections. In part (b) of the figure we see that scheme 2 outperforms others in terms of EDBP under all load. Comparing the throughput in scheme 3 and scheme 2, we observe little performance degradation in the throughput of scheme 2, only in heavy traffic. Part (c) provides the optimal retransmission probabilities for standard aloha when the throughput is maximized, which explains its corresponding large EDBP. In contrast, when the EDBP is minimized Aloha has optimal retransmission probabilities of around 0.1 in heavy load whereas all other versions have much higher retransmission probabilities. Part (d) shows the average delay, $(\mathrm{EDTP}+\mathrm{EDBP}) / 2$ of all the schemes.

An interesting observation that can be made by comparing scheme 2 and other MAC protocols such as CSMA/CA. In contrast to aloha and slotted Aloha, some other MAC protocols such as the CSMA/CA, used in IEEE 802.11, have the feature that a new arriving packet has to wait some (random) amount of time before attempting transmission, so as to avoid a possible collision. In our scheme 2, the impact of new arrivals on the system is similar to have such a random delay, since new arriving packets have less priority (power) and thus on transmitting upon arrival, they cannot cause collisions with a transmitted backlogged packet (if there is one). One can thus view this lower priority as generating a random delay which is, however, more flexible in our scheme since, unlike CSMA/CA, not all arriving packets have to wait a random time. At light load the arriving packets will wait less frequently than in heavy load since the probability of having a competing transmission at the same slot by a backlogged packet is smaller in light traffic.

Stability. In Figure 3(a), we illustrate the stability behavior for $q_{r}=0.15, q_{a}=$ $0.01, m=40, N=5$. The drift is the difference between the curves (representing the departure rate or $P_{\text {succ }}$ ) and the straight line representing the arrival rate $(m-n) q_{a}$. The system, although fluctuating, tends to move in the direction of the drift and consequently tends to cluster around the two stable points with rate excursions between the two (for scheme 1). Slotted Aloha is the only scheme that suffers from the bi-stability problem and the departure rate is at most $1 / e$ whereas for different power schemes it is quite higher. By choosing a large value of retransmission probabilities, we can obtain situations where schemes 1 and 2 acquire a bi-stable regime, and the scheme 3 remains stable for all values 


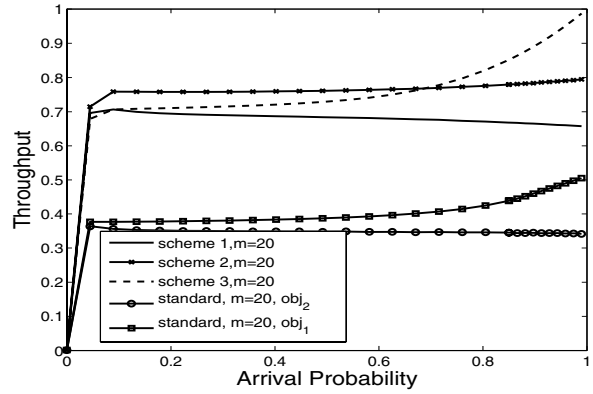

(a)

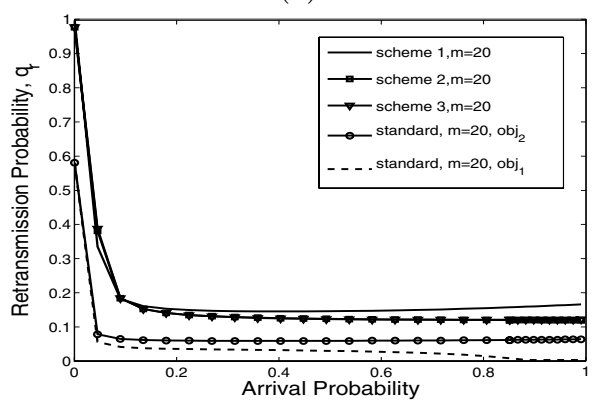

(c)

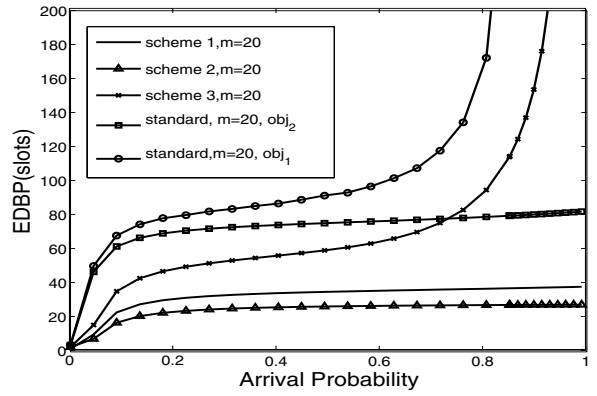

(b)

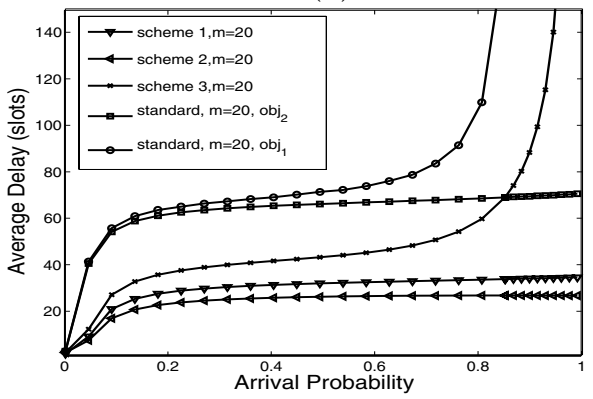

(d)

Fig. 2. (a), (b), (c) and (d) show the throughput, EDBP, optimal retransmission and the average delay respectively when the objective is to minimize EDBP, $o b j_{2} . o b j_{1}$ refers to the objective of maximizing the throughput. The number of mobiles is 20

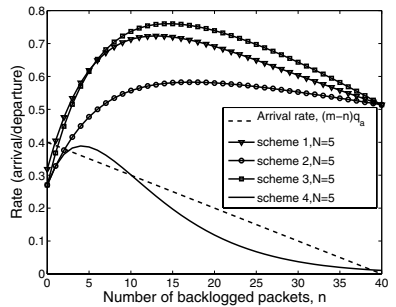

(a)

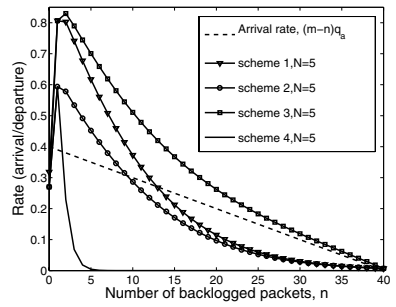

(b)

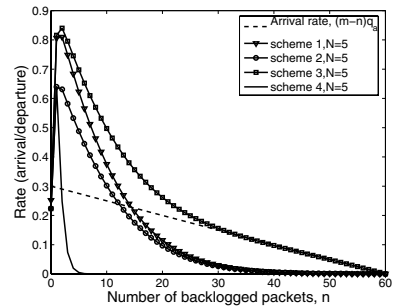

(c)

Fig. 3. Stability and instability of slotted Aloha schemes 1, 2, and 3: (a) $q_{a}=0.01$, $m=40, N=5$ and $q_{r}=0.15$. (b) $q_{a}=0.01, m=40, N=5$ and $q_{r}=0.8$, (c) $q_{a}=0.005, m=60, N=5$ and $q_{r}=0.8$ for all schemes

of retransmission probability $q_{r}$. For example, with 40 mobiles and $q_{a}=0.15$, scheme 1 and 2 suffer from the bi-stable problem when $q_{r}=0.8$ (see Figure 3 (b)). Note that the bi-stability can occur in all schemes, which is the case when the number of mobiles becomes large. For example, with 60 mobiles and $q_{a}=0.005$, the standard slotted Aloha is bi-stable already for $q_{r}=0.1$, Scheme 1 
Table 1. Average number of backlogged packets (ABP) and equilibrium point (s)

\begin{tabular}{|l|l|l|l|l|}
\hline schemes & same-power & no-prior & more-power & less-power \\
\hline ABP $q_{r}=0.1$ & 56.8 & 0.71 & 1.04 & 1.09 \\
\hline (un)stable eq· & $1.51,25.47$ & 0.67 & 1.449 & 1 \\
$q_{r}=0.1$ & 56.88 & & & \\
\hline ABP $q_{r}=0.5$ & 60 & 0.28 & 0.28 & 0.287 \\
\hline (un)stable eq. & $1.51,25.47$ & $0.136,28.85$ & $0.10,24.33$ & 0.208 \\
$q_{r}=0.5$ & 56.88 & 56.83 & 56.89 & \\
\hline ABP $q_{r}=0.9$ & 60 & 59.98 & 59.98 & 57.58 \\
\hline (un)stable eq. & $0.16,1.72$ & $0.07,12.43$ & $0.16,10.46$ & $0.11,26.70$ \\
$q_{r}=0.9$ & 60 & 59.98 & 59.98 & 56.99 \\
\hline
\end{tabular}

and 2 are bi-stable with $q_{r}=0.5$ and Scheme 3 becomes bi-stable with $q_{r}=0.95$ (see Fig 3(c)). Here, as well as in all other examples (not reported here) scheme 3 always turned out to have the largest region of parameters for which a unique stable point is obtained.

The average number of backlogged packets for (ABP) different schemes which correspond to their equilibrium points are given in Table 1 with $m=60, q_{a}=$ 0.005 and $N=5$. In the case of a single equilibrium, a good match is seen for schemes 1, 2 and 3, which means that the simple computation of the stable equilibrium can be used to approximate the expected number of backlogged packets. In standard Aloha we see that the congested stable equilibrium provides a very good approximation for the expected number of backlogged packets, which suggests that the system spends most of the time at that equilibrium.

We also observe same behavior in scheme 1 and 2 when the retransmission probability increases (around 0.5). Scheme 1 and 2 acquire bi-stable equilibrium with $q_{r}=0.5$. But contrary to standard aloha, we see from Table 1 that the expected number of backlogged packets for scheme 1 and 2 can be approximated by the desired stable equilibrium. Now if the mobiles become aggressive $\left(q_{r}\right.$ around 0.9 ), we see that the congested stable equilibrium provides a very good approximation for the expected number of backlogged packets in all schemes.

Multi-criteria Objective. Consider the objective given by $\alpha \operatorname{thp}(q)+(1-$ $\alpha) \frac{1}{D^{c}}, 0 \leq \alpha \leq 1$. This allows to handle QoS constraints: By varying $\alpha$ one can find appropriate tradeoff between the throughput and delays, so that the throughput be maximized while keeping the EDBP bounded by some constant. We plot the performance when $N=5$ and the number of mobiles is 20, for all the new power schemes in Figure 4 (a) and (b). We observe that scheme 3 (lesspower for retransmission) is insensitive to the value of $\alpha$ under different load. The optimal retransmission probabilities for scheme 3 are very close under both the objectives: when throughput is maximized and when EDBP is minimized. When $\alpha$ increases (i.e., gives more weight to throughput), the throughput of scheme 2 and scheme 1 increase with $\alpha$ at higher load. But the delay also increases for scheme 2 and scheme 1.

Game Problem: Maximizing individual Throughputs: We evaluate the performance of distributed game version of the problem considering throughput 


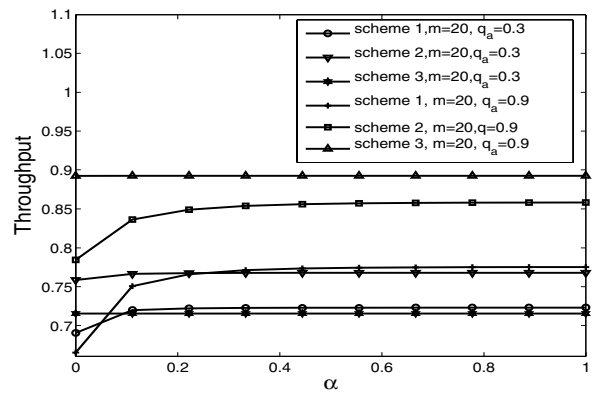

(a)

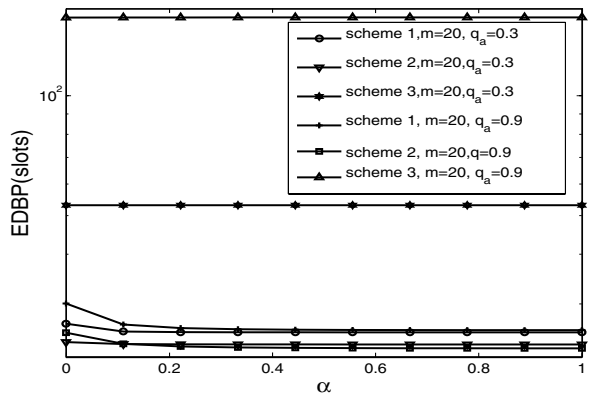

(b)

Fig. 4. (a) and (b) show throughput and delay of backlogged packets when the multicriteria objective is optimized for $N=5, m=20$

maximization.Fig 5 (a), (b) and (c) show the global equilibrium throughput (i.e. the expression in Eq. (9) times the number of mobiles), the equilibrium EDBP and the equilibrium retransmission probabilities, respectively. We observe that the performance of scheme 2 is the best in terms of EDBP. But at high load, the throughput of scheme 3 is best. The equilibrium retransmission probability is high (close to 1) and thus most aggressive for schemes 1-3 for any arrival probability. Standard aloha is less aggressive at equilibrium at light load. Possible explanation: If an individual tagged mobile were very aggressive in standard aloha (retransmission probabilities close to 1) then eventually all other mobiles would become backlogged which could increase the collision rate and thus decrease the throughput of the tagged mobile. Hence for some values of arrival probabilities the equilibrium behavior of standard aloha is not very aggressive. In contrast, the new schemes suffer less due to other mobiles becoming backlogged since they can reduce collisions due to the randomization and priorities. Hence increasing backlog of other mobiles does not penalize the tagged station anymore, so it has incentive to become more aggressive. The equilibrium transmission probabilities for schemes 1-3 are constants as function of $q_{a}$ given by 0.97 (for $m=4,10$ ). A remarkable feature of the new schemes is that the equilibrium throughput increases with $q_{a}$, as in the team problem. In contrast, for high loads the throughput decreases for scheme 1 and it also contains a decreasing behavior in standard Aloha. Thus the competition in the game formulation does not allow to benefit from increased input rates for standard aloha and scheme 1 (except for low values of $q_{a}$ ) whereas the new schemes do benefit from that. Finally, we note that schemes 1-3 all avoid the throughput collapse of standard aloha (for which we see in Fig. 5 (a) that the equilibrium throughput vanishes for both $m=10$ at $q_{a}>0.3$ and for $m=10$ at $\left.q_{a}>0\right)$.

Game Problem: Minimizing Individual EDBP. Next, we evaluate the performance of the distributed game problems of minimizing EDBP. We notice again from Figure 6(a) that the equilibrium throughput decreases with $q_{a}$ for scheme 


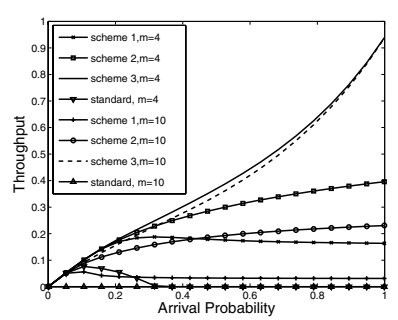

(a)

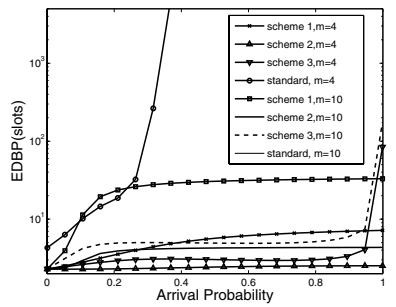

(b)

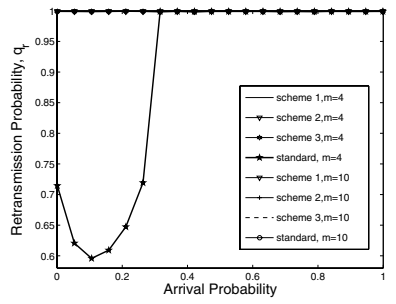

(c)

Fig. 5. (a), (b) and (c) show the throughput, EDBP and retransmission probability when the objective is to maximize the throughput for all the schemes for 4 and 10 mobiles and the number of power levels is 5

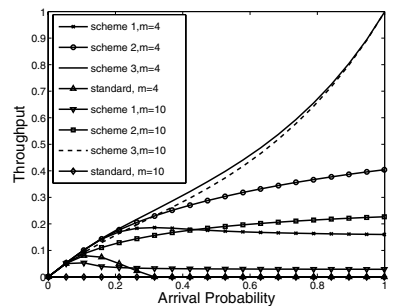

(a)

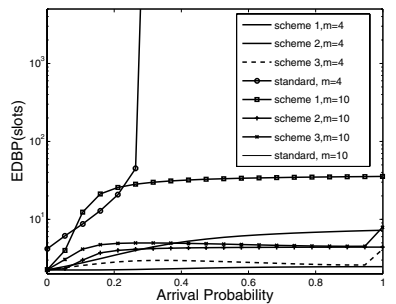

(b)

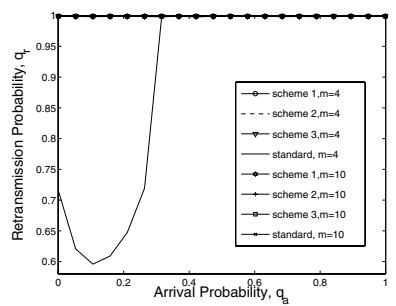

(c)

Fig. 6. (a), (b), and (c) show the throughput, EDBP and retransmission probability when the objective is to minimize the delay of backlogged packets for all the schemes for 4 and 10 mobiles and the number of levels is 5

1 (for arrival probabilities larger than 0.2) and for standard aloha (for $q_{a}>0$ ). In both schemes 2 and 3 it increases yet the increase is much larger in scheme 3 . This scheme outperforms all others for any $q_{a}$. Schemes 1-3 all avoid the throughput collapse of standard aloha. We observe a non-monotonic behavior of the equilibrium EDBP for scheme 3 in Figs 6(b). According to Eq. (11), this means that as the arrival rate increases, the throughput grows faster than the expected number of backlogged packets. Scheme 2 and 3 have very close EDBP which is better than scheme 1 and standard aloha for all $q_{a}$. we see that $1-3$ are very aggressive in terms of retransmission probabilities in Figs 6)(c). An interesting feature to note is that the throughput obtained when maximizing the individual throughput is less than that obtained when minimizing the EDBP. This is due to the fact that we are in a non-cooperative game setting, for which the equilibria are known not to be efficient (as is the case in the famous prisoner's dilemma paradox). 


\section{Conclusions}

We have studied two schemes that involve both prioritization as well as power diversity for increasing the throughput and decreasing the EDBP. We studied optimal choices of transmission probabilities both in a cooperative as well as in a non-cooperative setting. Scheme 3 has the best stability properties and the best throughput performance in the game setting. The throughput performance of schemes 2 and 3 benefit from increasing the arrival rate in the game scenario, in contrast with standard Aloha which suffers a throughput collapse, and with the power diversity scheme 1 (without priorities) whose equilibrium throughput decreases in high load. A remarkable feature of scheme 3 is that it performs very well in the game setting as compared to the team problem. In particular, when maximizing the throughput, we see that in heavy traffic it attains the maximum achievable throughput as is the case for the team formulation.

\section{References}

1. N. Abramson, "The Aloha system - another alternative for computer communications", AFIPS Conference Proceedings, Vol. 36, pp. 295-298, 1970.

2. E. Altman, D. Barman, A. Benslimane and R. El Azouzi, "Slotted Aloha with priorities and random power", http://www.inria.fr/mistral/personnel/Eitan. Altman/mobile.html

3. E. Altman, D. Barman, R El Azouzi and T. Jimenez, "A game theoretic approach for delay minimization in slotted aloha", ICC, 20-24, Paris, France, June 2004.

4. E. Altman, R El Azouzi and T. Jimenez, "Slotted Aloha as a Stochastic Game with Partial Information", WiOpt'03, Sophia Antipolis, France, March 3-5, 2003.

5. D. Bertsekas and R. Gallager, Data Networks, Prentice Hall, Englewood Cliffs, New Jersey, 1987.

6. Y. Jin and G. Kesidis, "Equilibria of a noncooperative game for heterogeneous users of an ALOHA network", IEEE Comm. Letters 6 (7), 282-284, 2002.

7. R. O. LaMaire, A. Krishna and M. Zorzi, "On the randomization of transmitter power levels to increase throughput in multiple access radio systems", Wireless Networks 4, pp 263-277, 1998.

8. A. B. MacKenzie and S. B. Wicker, "Selfish users in Aloha: A game theoretic approach", IEEE VTC, fall, 2001.

9. A. B. MacKenzie and S. B. Wicker, "Stability of Slotted Aloha with Multipacket Reception and Selfish Users," Infocom, April 2003.

10. J. J Metzner, On improving utilization in ALOHA networks, IEEE Transaction on Communication COM-24 (4), 1976.

11. L. G. Roberts, "Aloha packet system with and without slots and capture", Tech. Rep. Ass Note 8, Stanford Research Institute, Advance Research Projects Agency, Network Information Center, 1972.

12. J. H. Sarker, M. Hassan, S. Halme, Power level selection schemes to improve throughput and stability of slotted ALOHA under heavy load, Computer Communication 25, 2002.

13. Yalin E. Sagduyu and Anthony Ephremides, "Power Control and Rate Adaptation as Stochasic Games for Random Access", Proc. 42nd IEEE Conference on Decision and Control, Hawaii, Dec. 2003 